Wir suchen für unser Zentrales Forschungslabor in Heidelberg eine(n)

\title{
Metallographin/ Metallographen
}

Der Aufgabenbereich umfaßt die Untersuchung von Schutzschichten, Schweiß- und Lötnähten sowie Metallkeramik-Verbindungen. Farblabor, moderne Keramographie und metallographische Einrichtungen sind vorhanden.

Wir erwarten eine abgeschlossene Ausbildung bei einer entsprechenden Lehranstalt.

Von Vorteil aber nicht Bedingung, sind Kenntnisse und Erfahrungen auf den Gebieten HochtemperaturWerkstoffe und Keramographie.

Bitte senden Sie uns Ihre Bewerbungsunterlagen mit tabellarischem Lebenslauf, Zeugniskopien, möglichem Eintrittsdatum und Gehaltsvorstellung unter dem Kennzeichen ,ZFL' an unsere Abteilung Personal- und Sozialwesen.



BROWN, BOVERI \& CIE · AKTIENGESELLSCHAFT Geschäftsbereich Niederspannungsgeräte Eppelheimer Straße 82, 6900 Heidelberg 